\title{
High Prevalence of Imposterism Among Female Harvard Medical and Dental Students
}

\author{
Alison M. Holliday, MD, MPH' , Galina Gheihman, $\mathrm{MD}^{7}$, Cynthia Cooper, $\mathrm{MD}^{2}$, \\ Amy Sullivan, EdD ${ }^{3,4}$, Hiroe Ohyama, DMD, MMSc, PhD ${ }^{5}$, David E. Leaf, MD, MMSc 6 , and \\ Rebecca Karp Leaf, $M D^{7}$ (i)
}

'Department of Medicine, Brigham and Women's Hospital, Harvard Medical School, Boston, MA, USA; '2Department of Medicine, Massachusetts General Hospital, Harvard Medical School, Boston, MA, USA; ${ }^{3}$ Department of Medicine, Beth Israel Deaconess Medical Center, Harvard Medical School, Boston, MA, USA; ${ }^{4}$ Shapiro Institute for Education and Research, Beth Israel Deaconess Medical Center, Harvard Medical School, Boston, MA, USA; ${ }^{5}$ Department of Restorative Dentistry and Biomaterials Sciences, Harvard School of Dental Medicine, Boston, MA, USA; ${ }^{6}$ Division of Renal Medicine, Brigham and Women's Hospital, Harvard Medical School, Boston, MA, USA; ${ }^{7}$ Division of Hematology, Massachusetts General Hospital, Harvard Medical School, Boston, MA, USA.

J Gen Intern Med 35(8):2499-501

DOI: $10.1007 / \mathrm{s} 11606-019-05441-5$

(c) Society of General Internal Medicine 2019

$\mathrm{H}$ igh Prevalence of Imposterism Among Female Harvard Medical and Dental Students

David E. Leaf and Rebecca Karp Leaf contributed equally to this work.

\section{INTRODUCTION}

The imposter phenomenon (IP) is a psychological construct characterized by feelings of inadequacy and an irrational fear of being discovered as a "fraud" by colleagues and superiors. IP limits performance and is associated with anxiety, burnout, and low selfesteem across multiple professions and academic spheres. First described by psychologists Clance and Imes in 1978, IP was initially found to affect high-achieving women who believed that they were not as intelligent or capable as others believed. ${ }^{1}$

There is a small literature on IP in academic medicine and dentistry; however, robust data is lacking and existing studies are limited by small sample sizes or use of non-validated survey instruments. ${ }^{2,3}$

Here we report the largest study to date of IP among undergraduate medical and dental students, investigating risk factors for IP and focusing in particular on students with the highest levels of imposterism.

\section{METHODS}

Surveys were emailed to all Harvard Medical School (HMS) and Harvard School of Dental Medicine (HSDM) students $(n=753)$

Received September 7, 2019

Accepted September 26, 2019

Published online October 25, 2019 between June and August 2018 using a web-based program (Qualtrics). The study was deemed exempt by the Institutional Review Boards of HMS and Massachusetts General Hospital.

\section{IP Questionnaire}

Students were asked to complete the 20-question validated Clance Imposter Phenomenon Scale (CIPS), with responses scaled from 1 (not at all true) to 5 (very true). Higher scores on the CIPS are associated with greater feelings of imposterism. An example CIPS question is as follows: "I can give the impression that I'm more competent than I really am." In order to emphasize those respondents with the highest degree of imposterism, we defined IP as a score of 80 or higher, which corresponds to "intense IP" in the original publication of CIPS. ${ }^{1}$

\section{Statistical Analyses}

We used univariate and multivariable logistic regression to assess the association between student characteristics and IP. All comparisons were two tailed, with $P<0.05$ considered significant.

\section{RESULTS}

Demographic characteristics are shown in Table 1. Among the 753 students who received the survey, 485 completed all CIPS questions (64\%). The mean age of the respondents was 25 (range, 21 to 34) years. Of those that completed the CIPS questions, $37 \%$ were male, $52 \%$ were female, and $11 \%$ did not indicate their gender.

The mean CIPS score across all students was 65 (standard deviation, 13), with the distribution of IP scores according to male and female gender shown in Figure $1 \mathrm{a}$ and $\mathrm{b}$ respectively. Overall, $15 \%$ of students had a score of 80 or higher and thus met the criteria for intense IP. The prevalence of intense IP was $11 \%$ among males and $18 \%$ among females.] $\rightarrow$

Univariate predictors of IP included age (years, odds ratio [OR] $1.11,95 \%$ confidence interval $[\mathrm{CI}] 1.02-1.22$ ), female gender (OR 1.95, 95\% CI 1.15-3.31), and time off prior to medical 
a

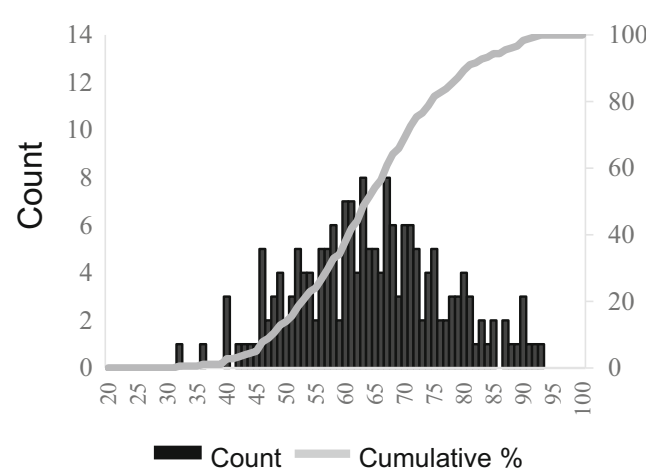

b

Women

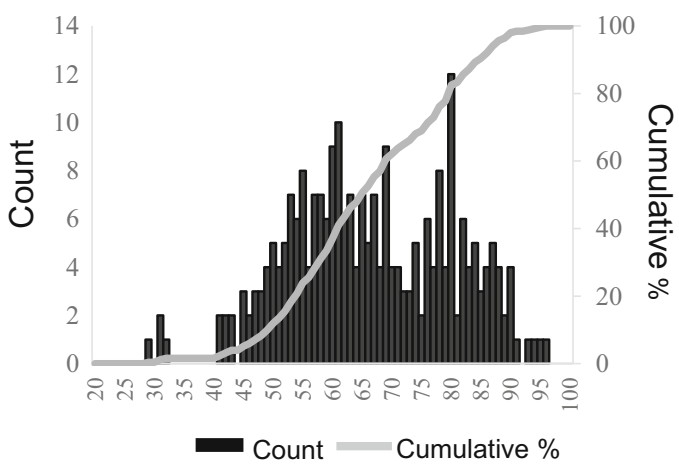

C Univariate predictors of IP

\begin{tabular}{|c|c|c|c|}
\hline Demographic Characteristics & & Odds Ratio $(95 \% \mathrm{Cl})$ & P-Value \\
\hline Age, years & $\boldsymbol{\varphi}$ & $1.11(1.02-1.22)$ & 0.02 \\
\hline Female gender & 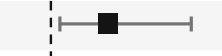 & $1.95(1.15-3.31)$ & 0.01 \\
\hline White race & $1 \boldsymbol{B}_{1}^{-1}$ & $0.81(0.50-1.32)$ & 0.40 \\
\hline Hispanic or Latino ethnicity & $\stackrel{1}{1}$ & $1.62(0.74-3.57)$ & 0.23 \\
\hline Family member is physician & $1+1$ & $0.92(0.54-1.56)$ & 0.76 \\
\hline \multicolumn{4}{|l|}{ Graduate School Characteristics } \\
\hline Time off before medical school & 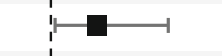 & $1.77(1.07-2.93)$ & 0.03 \\
\hline $\mathrm{PhD}$ track & $\longrightarrow$ & $1.56(0.77-3.14)$ & 0.22 \\
\hline Dental vs. Medical track & $\longmapsto$ & $1.00(0.48-2.49)$ & 0.99 \\
\hline Year at Harvard & p & $1.20(0.99-1.45)$ & 0.06 \\
\hline \multicolumn{4}{|l|}{ Future Plans } \\
\hline Surgical career plans & $H_{1}-1$ & $1.00(0.60-1.69)$ & 0.99 \\
\hline Strong desire for leadership & $\stackrel{1}{1}$ & $1.34(0.82-2.18)$ & 0.24 \\
\hline
\end{tabular}

\section{d Multivariate predictors of IP}

\begin{tabular}{|c|c|c|c|}
\hline Characteristic & & Odds Ratio $(95 \% \mathrm{Cl})$ & P-Value \\
\hline Age, years & i & $1.08(0.98-1.20)$ & 0.13 \\
\hline Female gender & 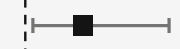 & $1.92(1.12-3.27)$ & 0.02 \\
\hline Time off before medical school & 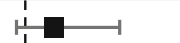 & $1.46(0.86-2.49)$ & 0.17 \\
\hline
\end{tabular}

Figure 1 a-d Female gender is a predictor of IP among Harvard medical and dental students. The distribution of CIPS scores among men (a) and women (b) is shown. The $X$-axes indicate the CIPS score and the $Y$-axes represent the number of students with a particular CIPS score, both total count (leftward $\boldsymbol{Y}$-axis) and cumulative percentage (rightward $\boldsymbol{Y}$-axis). Black bars indicate total raw count and gray lines depict cumulative percentage. Forest plots depict univariate (c) and multivariate (d) predictors of IP. CIPS, Clance Imposter Phenomenon Scale; IP, imposter phenomenon.

school (OR 1.77, 95\% CI 1.07-2.93) (Fig. 1c). In multivariable models, female gender remained the only independent predictor of intense IP (OR 1.92, 95\% CI 1.12-3.27; Fig. 1d).

\section{DISCUSSION}

The aim of this study was to investigate the prevalence and predictors of IP among HMS and HSDM students. We found that nearly $15 \%$ of all students in our sample scored above 80 on the CIPS scale, indicating severe imposterism. In multivariable-adjusted models, female gender was the sole significant predictor of intense IP, conferring a nearly twofold higher risk.

IP is pervasive within the health professions and likely under-reported. ${ }^{4}$ Although older studies have found a higher prevalence of IP among female medical and dental students, ${ }^{5}$ there is still much to be done in tackling this issue. Some 


\section{Table 1 Student Characteristics}

\begin{tabular}{ll}
\hline \hline Sociodemographic characteristics & \\
\hline Mean age, years (SD) & $25(3)$ \\
Male, $n(\%)$ & $179(37)$ \\
Race/ethnicity, $n(\%)$ & $205(46)$ \\
White & $158(36)$ \\
Asian & $20(5)$ \\
Black & $56(13)$ \\
Other ${ }^{\mathrm{a}}$ & $43(10)$ \\
Hispanic or Latino & $135(31)$ \\
Family member is a physician, $n(\%)$ & \\
Medical/dental school characteristics & \\
Track at Harvard, $n(\%)$ & $343(83)$ \\
Medical School & $72(17)$ \\
School of Dental Medicine & $48(11)$ \\
PhD & \\
Year at Harvard, $n(\%)$ & $106(24)$ \\
1st & $113(26)$ \\
2nd & $68(16)$ \\
3rd & $88(20)$ \\
4th & $60(14)$ \\
5th or greater & \\
Career characteristics & \\
College major, $n(\%)$ & $273(63)$ \\
(a) Biological or Physical Sciences & $109(25)$ \\
(a) Humanities, Social Sciences, or other & $53(12)$ \\
Double major from (a) and (b) above & $134(31)$ \\
Surgical Career Aspirations, $n(\%)$ & \\
\hline
\end{tabular}

Percentages are calculated on the basis of the number of students without missing data. Students could select multiple races/ethnicities. CIPS, Clance Imposter Phenomenon Scale; SD, standard deviation

"Includes students who indicated their race as "other" as well as students who identified as American Indian, Alaskan Native, or multiple races

${ }^{b}$ Students who elected to spend a fifth year completing research or an additional degree

authors have suggested that IP must be addressed on an organizational level: cultivating female mentors and role models and encouraging leadership training. ${ }^{6}$ Further, we suggest exploring individual factors that foster confidence and resilience. For instance, we might pursue additional analyses of those women without intense IP — what tools have they developed in order to avoid the pitfalls of severe imposterism? Finally, it is important to normalize IP, with frequent and open dialogue and discussion: too often trainees suffer in silence. Only by recognizing the weed of IP and addressing it at its source, we will be able to grow as a healthcare profession.

Acknowledgments: The authors thank Stephen Pelletier, $\mathrm{PhD}$, Department of Medicine, Beth Israel Deaconess Medical Center, Boston, MA; Shapiro Institute for Education and Research, Beth Israel Deaconess Medical Center, Boston, MA

Corresponding Author: Rebecca Karp Leaf, MD; Division of Hematology Massachusetts General Hospital, Harvard Medical School, 55 Fruit Street, Boston, MA 02114, USA (e-mail: Rkarp-Leaf@partners. org).

\section{Compliance with ethical standards:}

Conflict of Interest": The authors declare that they do not have a conflict of interest.

\section{REFERENCES}

1. Clance PR IS. The Impostor Phenomenon in High Achieving Women: Dynamics and Therapeutic Intervention. Psychotherapy Theory, Research and Practice 1978;15:241-7.

2. Villwock JA, Sobin LB, Koester LA, Harris TM. Impostor syndrome and burnout among American medical students: a pilot study. Int J Med Educ 2016;7:364-9.

3. Hu KS, Chibnall JT, Slavin SJ. Maladaptive Perfectionism, Impostorism, and Cognitive Distortions: Threats to the Mental Health of Pre-clinical Medical Students. Acad Psychiatry 2019.

4. LaDonna KA, Ginsburg S, Watling C. "Rising to the Level of Your Incompetence": What Physicians' Self-Assessment of Their Performance Reveals About the Imposter Syndrome in Medicine. Acad Med 2018;93:763-8.

5. Henning K, Ey S, Shaw D. Perfectionism, the imposter phenomenon and psychological adjustment in medical, dental, nursing and pharmacy students. Med Educ 1998;32:456-64.

6. Mullangi S, Jagsi R. Imposter Syndrome: Treat the Cause, Not the Symptom. JAMA 2019;322:403-4.

Publisher's Note Springer Nature remains neutral with regard to jurisdictional claims in published maps and institutional affiliations. 